\title{
Regional Lymph Node Dissection
}

National Cancer Institute

\section{Source}

National Cancer Institute. Regional Lymph Node Dissection. NCI Thesaurus. Code C48935.

Surgical removal of the lymph nodes located in a particular anatomic region. This procedure is commonly performed as a component of cancer staging. 